\title{
Three-Dimensional Structure of Turbulent Flow in Mixing T-Junction*
}

\author{
Masafumi HIROTA* ${ }^{* *}$, Hideo ASANO ${ }^{* * *}$, Hiroshi NAKAYAMA****, \\ Taichi ASANO ${ }^{\dagger}$ and Shunsaku HIRAYAMA***
}

\begin{abstract}
Experimental results on the turbulent mixing of hot and cold airflows in a T-junction are reported, which simulates the HVAC unit used in an automobile air conditioning system. Experiments are conducted keeping Reynolds number and temperature of the main flow at $2.5 \times 10^{4}$ and $12^{\circ} \mathrm{C}$, respectively, and the velocity of the branch flow $\left(60^{\circ} \mathrm{C}\right)$ is changed for three velocity ratios of $0.5,1$ and 2 . The flow from the branch is separated at the edge of the T-junction and forms a large separation bubble. Longitudinal vortices are formed around this separation bubble, thus the flow field has a three-dimensional structure. In spite of such a complex flow field, the mean temperature in the thermal mixing layer shows quite uniform distributions in the spanwise direction, and the strong turbulence produced around the separation bubble does not work effectively to the thermal mixing of hot and cold airflows.
\end{abstract}

Key Words: Turbulence, Three-Dimensional Flow, Turbulent Mixing, Heat Transfer, Flow Measurement, T-Junction

\section{Introduction}

In thermal equipments, a T-junction in which two flows with different velocities and temperatures are mixed turbulently is often encountered: one of the typical mixing T-junction can be found in the HVAC (Heating, Ventilating, Air-Conditioning) unit used for an automobile air-conditioning system ${ }^{(1)}$. Figure 1 shows a schematic diagram of the HVAC unit. All air taken by the fan is once cooled to $5^{\circ} \mathrm{C}$ by the evaporator to reduce humidity, and a part of this cold air is fed into the heatercore and is heated to $80^{\circ} \mathrm{C}$. Then, they are mixed together at appropriate flow-rate ratios to control the temperature of air blown into the cabin. The flow-rate ratio of hot and

* Received 2nd March, 2006 (No. 05-0225). Japanese Original: Trans. Jpn. Soc. Mech. Eng., Vol.71, No.712, B (2005), pp.2970-2978 (Received 3rd March, 2005)

** Graduate School of Engineering, Nagoya University, Chikusa-ku, Nagoya 464-8603, Japan.

E-mail: hirota@mech.nagoya-u.ac.jp

*** Air-Conditioning Testing and Evaluation Department, DENSO CORPORATION, Showa-cho, Kariya 448-8661, Japan

**** Fuel Cell Laboratory, Daido Institute of Technology, Minami-ku, Nagoya 457-8530, Japan

$\dagger$ Air-Conditioning R\&D Department 3, DENSO CORPORATION, Showa-cho, Kariya 448-8661, Japan cold air is determined by the opening of the air-mix door that is located between two heat exchanges ${ }^{(1)}$. Thus, in the HVAC unit, the hot and cold airflows impinge at nearly right angles with various velocity ratios depending on the air temperature required in the cabin. This situation can be regarded as the turbulent thermal mixing of air in the T-junction.

Nowadays it is desired that HVAC unit be designed virtually by making use of CAE to abridge the design process. This virtual design requires numerical simulations of turbulent thermal mixing of hot and cold air. At the present stage, however, the reliability of the calculated

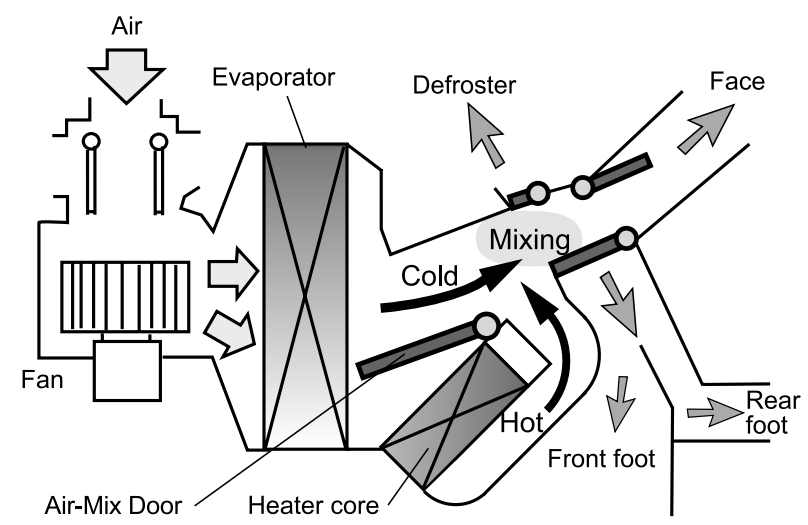

Fig. 1 Schematic diagram of automobile HVAC unit 


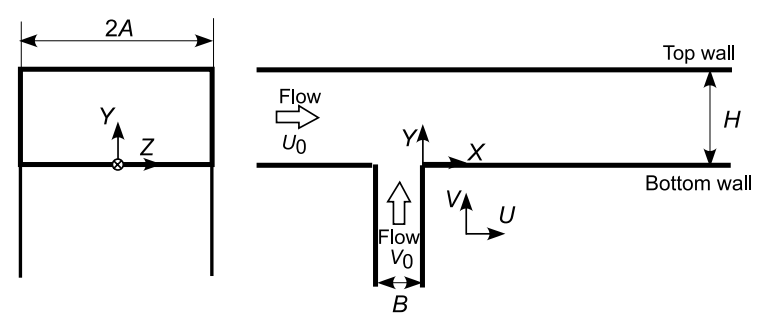

Fig. 2 Test channel $(A R=2)$

velocity and temperature distributions in the mixed airflow is not high enough to be directly applicable to the design of the HVAC unit. This is because the flow field in the Tjunction shows considerably complex features accompanied by the flow separation and reattachment, secondary flow, anisotropy of turbulent stresses, etc., and the present turbulence models cannot predict such complex flows with sufficient reliability. In addition to turbulence models, the modeling of the turbulent heat fluxes in such a complex flow is also a key issue for improving the reliability of numerical simulations of the turbulent thermal mixing in the HVAC unit ${ }^{(1)}$. Although detailed experimental data are indispensable to the development and evaluation of the turbulence and turbulent heat flux models, they are quite scarce now even in relatively simple flow geometries.

Based on these points, in this study, the mixing zone in the HVAC unit has been modeled by a T-junction with rectangular cross sections as shown in Fig. 2, and the velocity and mean temperature distributions in it have been measured taking the aspect ratio of the channel and the velocity ratio of hot and cold air as experimental parameters. It should be noted that this kind of flow geometry has been investigated extensively to date in relation to the film cooling of a gas turbine blade and the thermal striping in a nuclear power plant ${ }^{(2)-(6)}$. In those researches, the width (spanwise length) of the branch is smaller than that of the main channel. In the present case, however, the width of the branch is equal to that of the main channel (see Fig. 2). This is a geometrical feature of the HVAC unit, but there are only few papers that treat this kind of T-junctions; moreover, it is usually assumed that the flow field is two-dimensional in this type of T-junctions ${ }^{(7)}$. In this study, we make clear the three-dimensional structure of the flow field in the T-junctions using PIV and examine its influence on the mean temperature distributions of the mixed flow.

\section{Nomenclature}

$A$ : half of the spanwise length of the main channel cross section

$A R:$ aspect ratio of the main channel $=2 A / H$

$B: X$-directional length of the branch cross section

$H$ : height of the main channel cross section

$L$ : reattaching length

$T$ : time-averaged local temperature of mixed flow
$T_{c}$ : initial temperature of cold airflow (main-channel flow)

$T_{h}$ : initial temperature of hot airflow (branch flow)

$\Delta T$ : initial temperature difference between hot flow and cold flow $=T_{h}-T_{c}$

$U, V, W$ : time-averaged velocity components in the $X-, Y$ and $Z$-directions

$U_{0}, V_{0}$ : mean velocities in the main channel and the branch before the flow merging

$u, v, w$ : fluctuating velocity components in the $X-, Y$ - and $Z$-directions

$u^{\prime}, v^{\prime}, w^{\prime}$ : root-mean-square of $u, v$ and $w$, for example $u^{\prime}=$ $\sqrt{\overline{u^{2}}}$

$V R:$ velocity ratio $=V_{0} / U_{0}$

$X, Y, Z$ : coordinate system (see Fig. 2)

\section{Experimental Apparatus and Procedure}

The test channel is illustrated in Fig. 2 together with the coordinate system. The airflows in the main channel (horizontal channel) and the branch (vertical channel) correspond to the cold and hot flows in the HVAC unit, respectively. They are mixed in the T-junction after flowing through the heat exchangers, the settling chambers, and flow nozzles. The aspect ratio of the main channel ( $A R$ $=$ width $2 A$ /height $H$ ) was changed as 1,2 and 3 based on the practical HVAC unit. In this study, the height of the main channel $H$ was kept at $40 \mathrm{~mm}$ and the width $2 A$ was changed as $40 \mathrm{~mm}, 80 \mathrm{~mm}$ and $120 \mathrm{~mm}$ for $A R=1$, 2 and 3, respectively. As to the branch, the width was the same as that of the main channel and this is a geometric feature of the HVAC unit. The "height" ( $X$-way length) $B$ was kept at $20 \mathrm{~mm}$; namely, the cross section of the branch was $40 \mathrm{~mm} \times 20 \mathrm{~mm}, 80 \mathrm{~mm} \times 20 \mathrm{~mm}$, and $120 \mathrm{~mm} \times 20 \mathrm{~mm}$ for $A R=1,2$, and 3, respectively. Experiments were conducted keeping the Reynolds number of the main-channel flow at $2.5 \times 10^{4}$. The velocity of the branch flow $V_{0}$ was determined so that the velocity ratio $V R=V_{0} / U_{0}$ be changed as $0.5,1.0$ and 2.0, where $U_{0}$ is the mean velocity of the main-channel flow.

The velocity distributions were measured with a 2-D PIV system under an isothermal condition. The flow was seeded by oil particles ( $\sim 5 \mu \mathrm{m}$ in diameter) provided from a Laskin-nozzle type particle generator. The captured particle images were processed by a direct cross-correlation code developed in our laboratory with sub-pixel accuracy. For the statistical analysis, the mean velocity and the intensities of the velocity fluctuations were computed by averaging 500 instantaneous velocity fields. The measurements were made in the $X-Y$ planes of $Z / A=0 \sim-0.875$, and in the $Y-Z$ planes of $X / B=0 \sim 5$ to clarify the threedimensional structure of the flow field. The downstream end of the measuring region $(X / B=5)$ was determined based on the size of the mixing zone in a real HVAC unit.

The time-averaged local temperature distributions of 
the mixed flow were measured by a thermocouple rake. The temperature of the main-channel flow $T_{c}$ and that of the branch flow $T_{h}$ were set at $12^{\circ} \mathrm{C}$ and $60^{\circ} \mathrm{C}$, respectively, and the test channel was covered by heat insulators in this experiment. In order to avoid the influences of the thermocouples on the velocity field, the test channel was magnified by a factor of 1.5 in comparison with that for the PIV measurement. Thus, the main channel in which the mean temperature distributions were measured had a cross section of $120 \mathrm{~mm} \times 60 \mathrm{~mm}$ for $A R=2$. The Reynolds number of the main-channel flow is $2.5 \times 10^{4}$; in this case the Richardson number is about $3 \times 10^{-3}$. This value is equivalent to that of a real HVAC unit, and the influence of the buoyancy force is negligibly small. Indeed, we confirmed that the velocity distributions measured under the isothermal condition agreed well with those obtained with $T_{h}=60^{\circ} \mathrm{C}$ and $T_{c}=12^{\circ} \mathrm{C}$.

Figure 3 shows examples of the $Y$-way distributions of the mean velocity $U$, RMS of the fluctuating velocity component $u^{\prime}$ and mean temperature $T$ measured in the symmetric plane of the channel $(Z / A=0)$ at $X / B=-3$. Both the velocity and mean temperature show uniform distributions over the region covering about $80 \%$ of channel height $H$. In this study, we changed the conditions of the main and branch flows before the T-junction by placing several kinds of wire-meshes and perforated plates at its inlets, but noticeable influences were not observed in the characteristics of the velocity distributions after the flow merging. This means that the flow field after the Tjunction is quite insensitive to the inlet conditions of the main and branch flows.

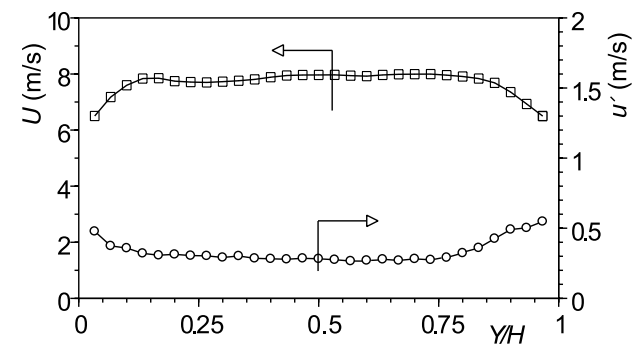

(a) Mean velocity $U$ and turbulent intensity $u^{\prime}$

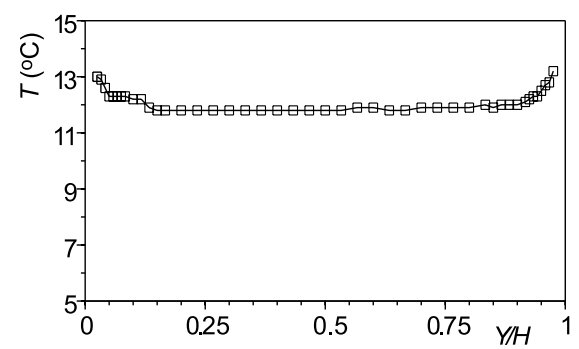

(b) Mean temperature $T$

Fig. 3 Inlet conditions of main channel flow at $X / B=-3(A R=$ $2, V R=1)$

\section{Results and Discussion}

\subsection{Overview of flow}

Figure 4 (a) - (c) shows the mean velocity vector diagrams for $V R=0.5,1$ and 2, respectively, measured in the symmetric plane of the channel $(Z / A=0)$ with $A R=2$. The time-averaged interface between the main-channel flow and the branch flow, which is obtained by the flow visualization, is shown in each figure by a broken line. The flow entering the main channel from the branch is separated at the downstream edge of the T-junction, and a large separation bubble is formed along the bottom wall of the main channel. As $V R$ is increased, the separation bubble is extended in the $X$-direction. The solid triangle in Fig. 4 shows the $X$-coordinate at which $U / U_{0}$ measured at $Y / H=0.06$ (measuring point nearest the bottom wall) changes its sign. This location is regarded as the reattaching point of the flow that is separated at the downstream edge of the T-junction ${ }^{(8)}$, and the distance between $X / B=0$ and this $X$-coordinate is defined as the reattaching length $L$. Figure 5 shows the variation of $L / B$ against $A R$ and $V R$. $L$ increases as $V R$ is increased but it is quite insensitive to $A R$. This suggests that the velocity field is much influenced by $V R$ but the influence of $A R$ is less significant.

\subsection{Secondary flow}

The secondary flow vectors measured in four cross sections at $V R=1$ are shown in Fig. 6. As observed in Fig. 4 , the cross sections at $X / B=1$ and 2 correspond to the locations at which the separation bubble develops in

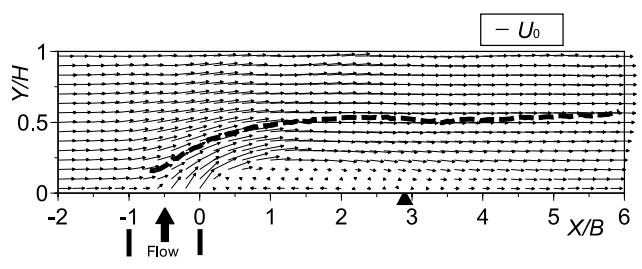

(a) $V R=0.5$

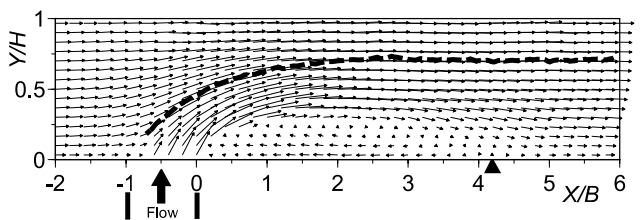

(b) $V R=1$

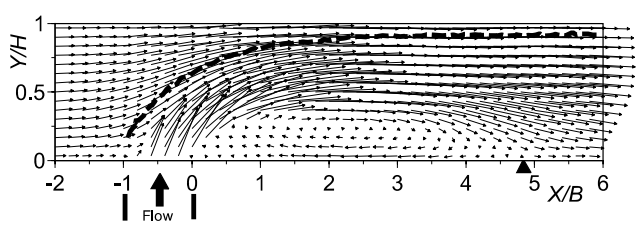

(c) $\quad V R=2$

Fig. 4 Mean velocity vectors in symmetric plane $(Z / A=0$, $A R=2$ ) 


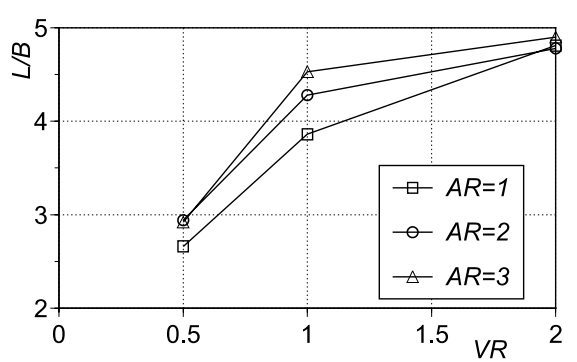

Fig. 5 Variation of reattaching length in $Z / A=0$ against $A R$ and $V R$
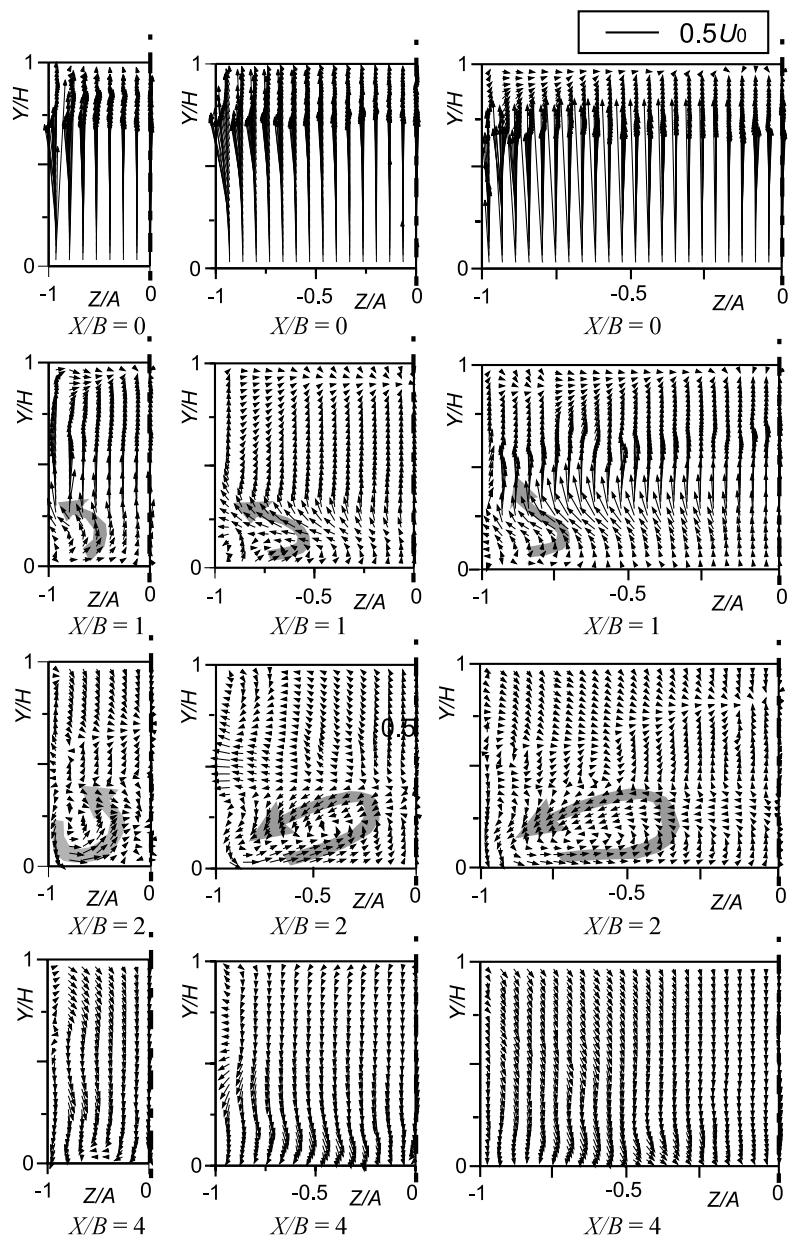

(a) $A R=1$

(b) $A R=2$

(c) $A R=3$

Fig. 6 Secondary flow vectors $(V R=1)$

the $Y$-direction, and the location of $X / B=4$ corresponds to the flow reattaching point. The results obtained in a half cross section of $Z / A<0$ are shown here.

At $X / B=0$, the upward currents are dominant over the cross section of the main channel. This upward flow becomes weaker at $X / B=1$ and 2, and a longitudinal vortex rotating in a counter-clockwise direction can be observed near the lower corner at $X / B=2$ for all $A R$. It is estimated that this longitudinal vortex is generated by the centrifugal force that arises due to the curvature of the streamlines of the flow entering from the branch. This

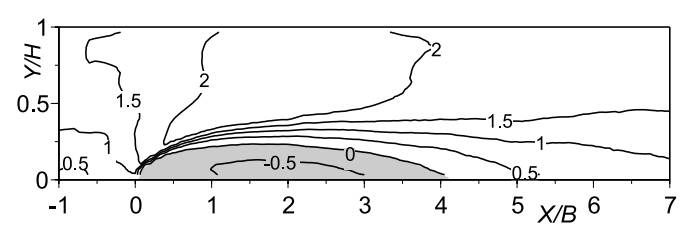

(a) $Z / A=0$

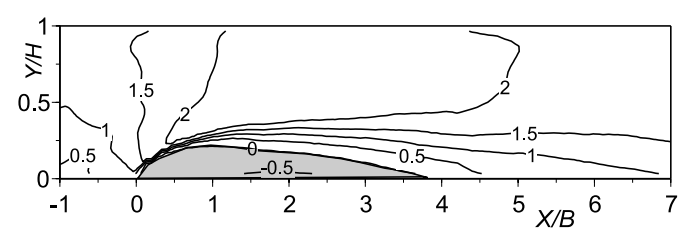

(b) $Z / A=-0.5$

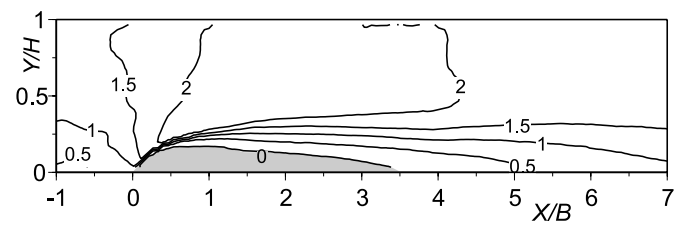

(c) $Z / A=-0.75$

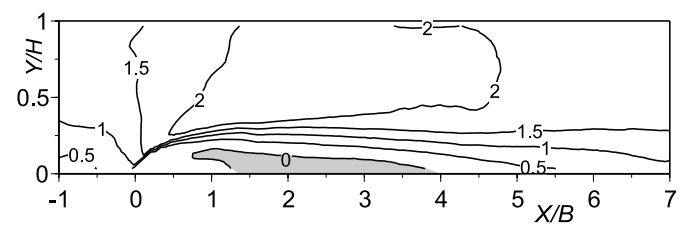

(d) $Z / A=-0.875$

Fig. 7 Spanwise variation of $U / U_{0}(A R=2, V R=1)$

mechanism of vortex generation is similar to that in a flow through a 90-deg bend ${ }^{(9)}$. At $X / B=4$, the longitudinal vortex disappears because the curvature of the streamline becomes quite small as recognized from Fig. 4 (b).

As $A R$ is increased, the longitudinal vortex at $X / B=2$ is stretched in the spanwise direction. However, the qualitative features of the secondary flow, such as rotating direction of the longitudinal vortices, do not change depending on $A R$. Moreover, as recognized from Fig. 5, the flow reattaching point is not influenced by $A R$. These results suggest that, under the present experimental conditions, characteristics of the flow field in a channel with some $A R$ can be estimated from those obtained for a different $A R$. Thus, in the following, the results for $A R=2$ are presented.

\subsection{Three-dimensional structure of separation bubble}

The spanwise variation of the primary flow velocity $U$ is examined to make clear the three-dimensional characteristics of the flow field, directing a special attention to the configuration of the reverse flow region. Figure 7 shows the contour maps of $U / U_{0}$ for $V R=1$ measured at different $Z / A$. The gray regions correspond to the reverse flow of $U / U_{0}<0$. It is observed that, as the sidewall is approached (increasing $|Z / A|$ ), the height ( $Y$-way scale) of the reverse flow region becomes smaller and the reattach- 


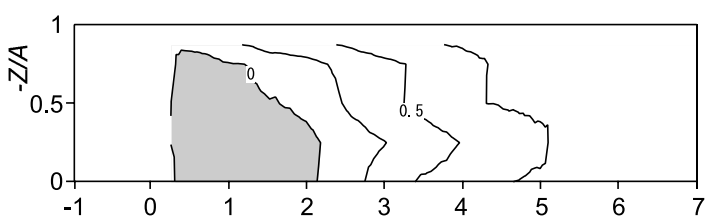

(a) $V R=0.5$

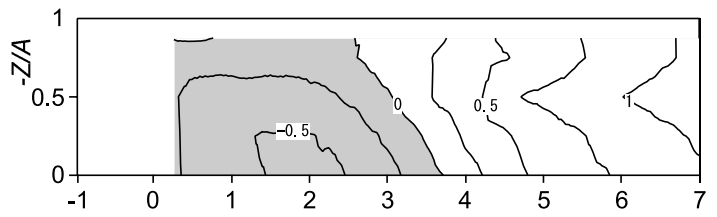

(b) $\quad V R=1$

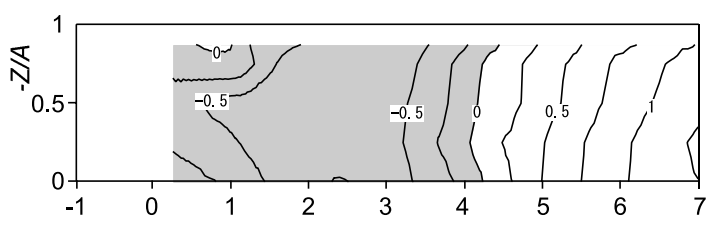

(c) $V R=2$

Fig. 8 Distributions of $U / U_{0}$ in the plane of $Y / H=0.1$

ing point moves upstream.

Figure 8 is a contour map of $U / U_{0}$ obtained in the plane of $Y / H=0.1$ that is located near the bottom wall of the main channel. As expected from Fig. 7, the trailing edge of the reverse flow region is stretched further downstream near the spanwise centerline for $V R=0.5$ and 1. It is recognized that the separation bubble has a complex three-dimensional shape. As recognized from a comparison of Figs. 6 and 7, the longitudinal vortex formed near the lower corner at $X / B=2$ is located as it straddles the boundary between the reversed flow region and the favorable flow region outside it. Thus, it contributes to the momentum exchange between the favorable flow region and the reverse flow region. Moreover, the separation of the flow is suppressed near the sidewall due to the effect of the viscous force. These influences of the momentum exchange by the secondary flow and of the viscous force causes such a complex configuration of the separation bubble. It is found that the spanwise variation of the longitudinal scale of the reverse flow region becomes smaller at higher $V R$.

\section{4 Turbulence intensities}

Figure 9 (a) and (b) shows the contour maps of the turbulence intensities $u^{\prime} / U_{0}$ and $v^{\prime} / U_{0}$ measured in $Z / A=0$ at $V R=1$. In these maps, the $Y$-coordinate of the maximum mean temperature gradient $\partial T / \partial Y$ at each $X / B$, i.e., the vertical center of the thermal mixing layer, is illustrated by a solid line, together with the boundary of the reverse flow region. The values of $u^{\prime} / U_{0}$ are very large around the shear layer formed outside the reverse flow region, and the high $u^{\prime}$ region develops in the $Y$-direction as the flow proceeds downstream. On the other hand, the vertical velocity component $v^{\prime}$ shows the local maximums not

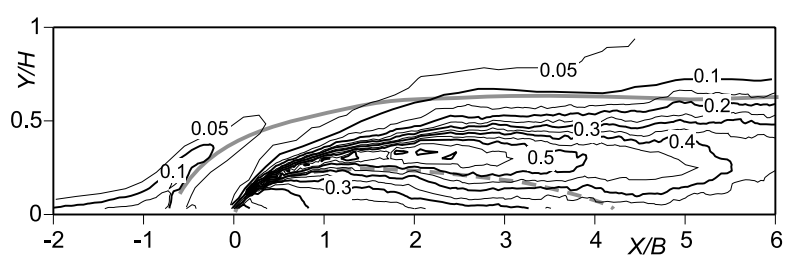

(a) $u^{\prime} / U_{0}$

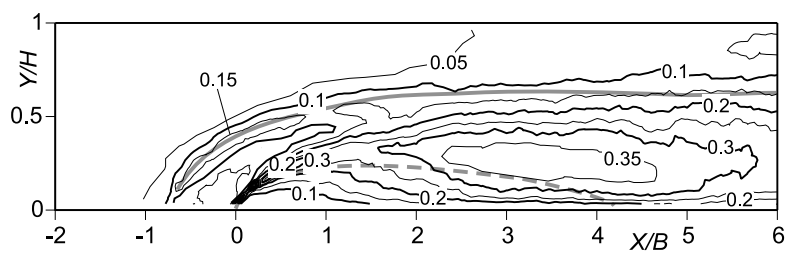

(b) $v^{\prime} / U_{0}$

Fig. 9 Distributions of turbulence intensities in $Z / A=0(V R=$ 1)

only in the shear layer around the separation bubble but also in the mixing layer near the flow merging region of $X / B=-1 \sim 1$. In this region, as recognized from Fig. 4 , the flow from the branch impinges on the main-channel flow and it is curved in the $X$-direction. As a result, the velocity gradients $\partial V / \partial X$ and $\partial V / \partial Y$ increase near the interface of two flows and the production of $v^{\prime}$ is promoted. This is a reason for high $v^{\prime}$ in the mixing layer. This large $v^{\prime}$ contributes directly to the turbulent transport of heat between the hot and cold flows. In the further downstream region of $X / B>2$, the increase of $v^{\prime}$ around the separation bubble is predominant and the local maximum of $v^{\prime}$ in the mixing layer becomes indistinctive. It is thought that the increase of $v^{\prime}$ around the separation bubble is mainly caused by the redistribution from $u^{\prime}$.

Next, in Fig. 10, the contour maps of $v^{\prime} / U_{0}$ obtained in several cross sections (half cross section of $Z / A<0$ ) in the main channel for three $V R$ are presented. In case of $V R=0.5$ and 1 , the high $v^{\prime}$ region observed near the bottom wall that corresponds to the separation bubble shows uniform distribution in the spanwise direction just after the flow merging. In the downstream region of $X / B>2$, however, it shows a three-dimensional feature in which $v^{\prime}$ shows higher values near the $Y$-axis. This distribution of $v^{\prime}$ is consistent with that of the separation bubble, which has a three-dimensional structure with a longer trailing edge near the spanwise centerline of the bottom wall. As described above, the 3-D feature of the separation bubble appears more clearly at lower velocity ratio. Therefore, the spanwise variation of $v^{\prime}$ is also pronounced under the relatively low $V R$ condition. On the other hand, in the mixing layer located above the separation bubble, $v^{\prime}$ is distributed quite uniformly in the spanwise direction at any $X / B$. Thus, it follows that the longitudinal vortex and the separation bubble, which bring about 3-D distribution of $v^{\prime}$ near the bottom wall, does not exert serious influences on the $v^{\prime}$ distribution in the mixing layer. 

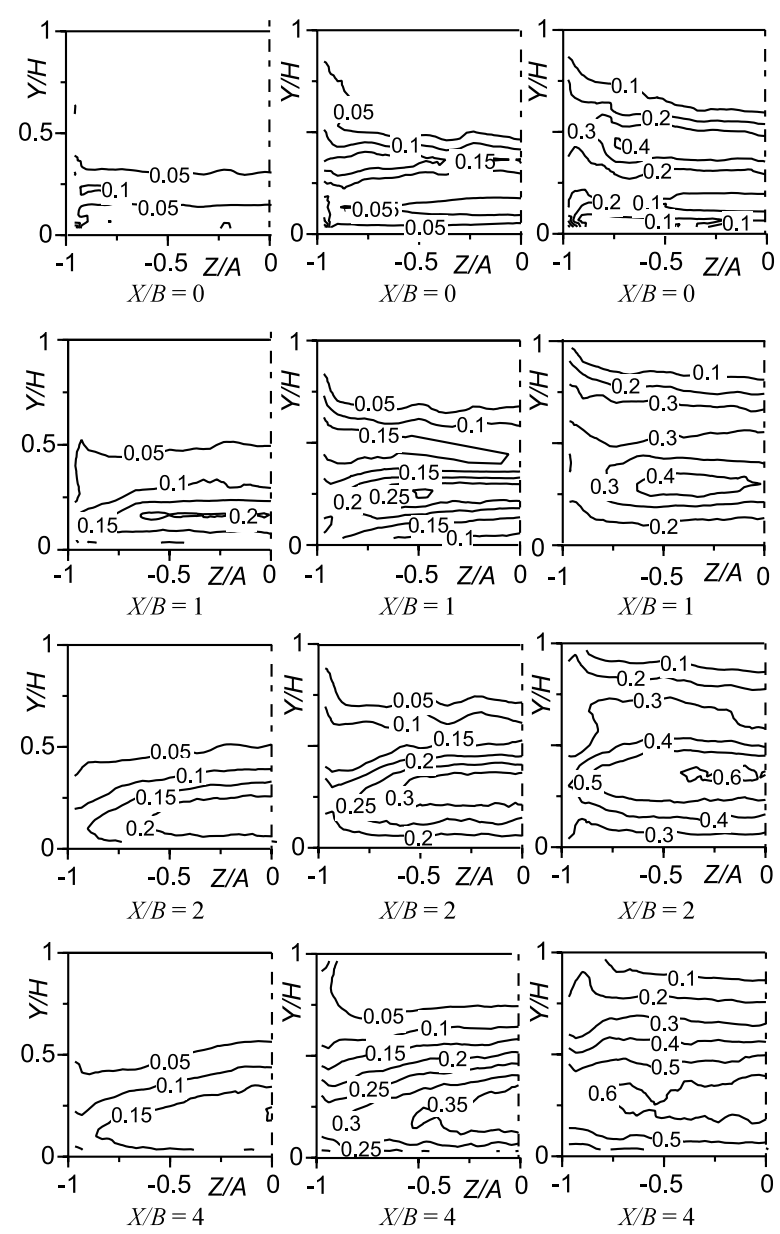

$\begin{array}{lll}\text { (a) } V R=0.5 & \text { (b) } V R=1 & \text { (c) } V R=2\end{array}$

Fig. 10 Distributions of $v^{\prime} / U_{0}$ with various $V R$

The distributions of the spanwise fluctuating velocity component $w^{\prime}$ are not presented here due to the limitation of the space. It was observed that the values of $w^{\prime}$ in the mixing layer were somewhat smaller than those of $v^{\prime}$, but in the shear layer around the separation bubble the distributions of $w^{\prime}$ agreed quantitatively well with those of $v^{\prime}$.

\subsection{Mean temperature distributions}

The contour maps of the mean temperature normalized by the initial temperature difference of hot and cold flow $\left(T-T_{c}\right) / \Delta T$ are shown in Fig. 11. The measured cross sections are the same as those of $v^{\prime}$ maps. Here the thermal mixing layer is defined as the region in which the mean temperature gradient $\partial T / \partial Y$ has non-zero values.

As described above, the flow field after the flow merging has a complex three-dimensional structure accompanied by a longitudinal vortex. In Fig. 11, however, the contours of the mean temperature are distributed uniformly in the spanwise direction at all $X / B$, and thus they do not reflect the three-dimensional feature of the flow field. This is understood as follows from a comparison of Figs. 6(b) and $11(\mathrm{~b})$. The longitudinal vortex that brings about the three-dimensional flow structure is confined inside the hot flow region with constant temperature that is located be-
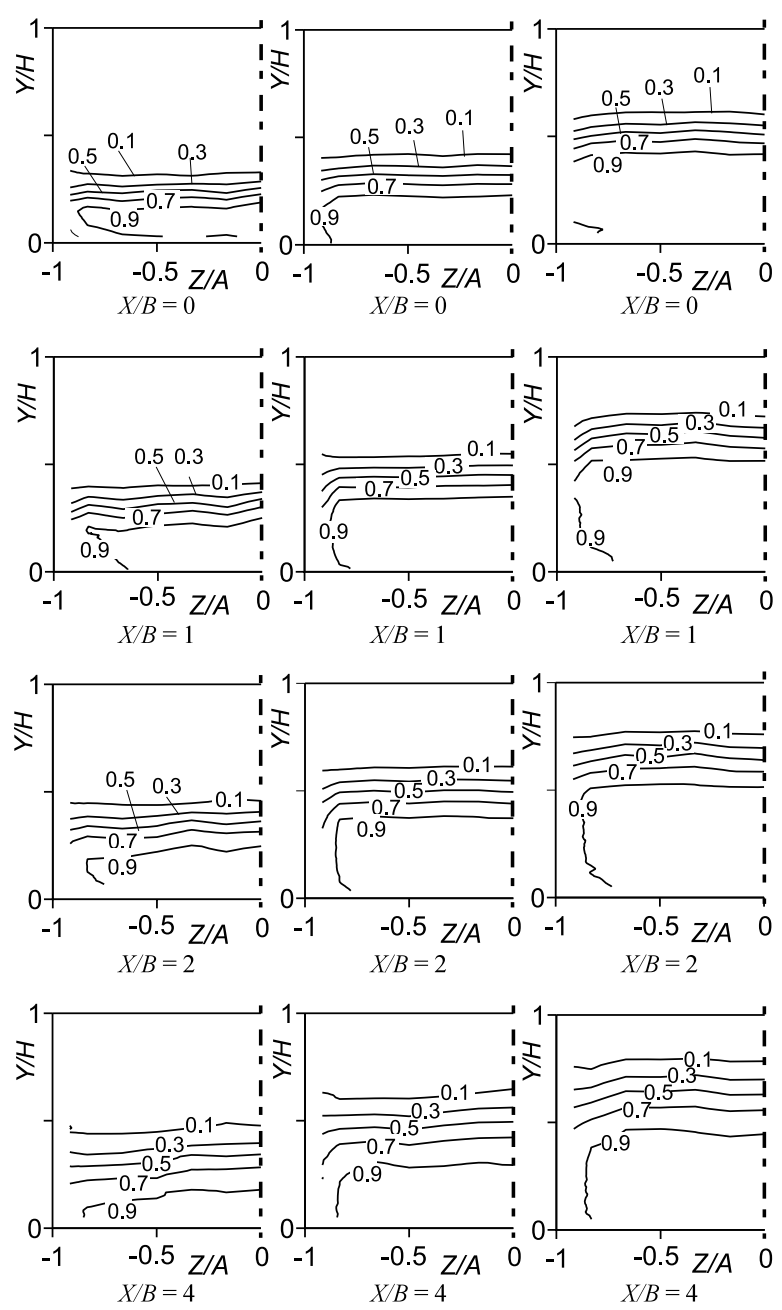
(a) $V R=0.5$
(b) $V R=1$
(c) $\quad V R=2$

Fig. 11 Distributions of mean temperature $\left(T-T_{c}\right) / \Delta T$ with various $V R$

low the mixing layer, and thus it does not exert direct influence upon the temperature distribution in the mixing layer. This result is consistent with the distribution of $v^{\prime}$, which is distributed uniformly in the spanwise direction in the mixing layer in spite of the three-dimensional feature of the separation bubble.

It is also found from Fig. 11 that the upper boundary of the thermal mixing layer hardly changes its height in the streamwise direction in $X / B>2$, although the mixing layer develops gradually downward in its lower part. In order to explain the reason for such an asymmetric development of the thermal mixing layer in the $Y$-direction, we compare the distributions of $v^{\prime}$ and $\partial T / \partial Y$, taking into consideration that the main production of the turbulent heat flux $\overline{v t}$, which contributes directly to the mixing of the hot and cold airflows, is expressed by the following equation.

Production of $\overline{v t} \cong \overline{v^{2}} \frac{\partial T}{\partial Y}$

Figure 12 shows the result of this comparison ob- 


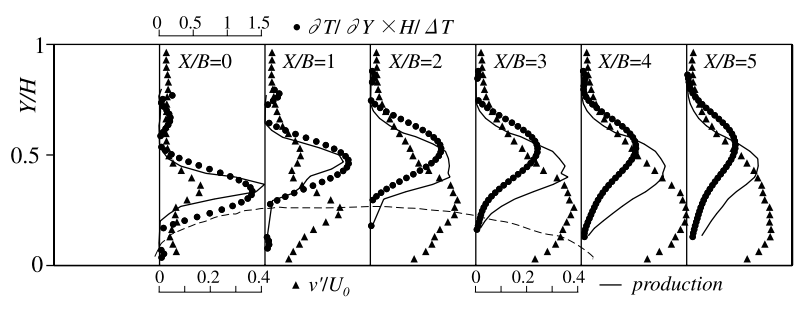

Fig. 12 Distributions of mean temperature gradient $\partial T / \partial Y$, turbulence intensity $v^{\prime}$ and production of $\overline{v t}$

tained in $Z / A=0$ and for $V R=1$. The boundary of the reverse flow region (broken line) and the distributions of the production term expressed above (solid line) are also shown in this figure. As previously mentioned, the velocity fluctuation $v^{\prime}$ shows the local maximum in the mixing layer near the flow merging region $(X / B=0$ and 1$)$. In the downstream region of $X / B>2$, however, the increase of $v^{\prime}$ in the shear layer around the separation bubble becomes predominant and the local maximum of $v^{\prime}$ in the mixing layer disappears. On the other hand, $\partial T / \partial Y$ attains the local maximums in the vertical center of the thermal mixing layer at any $X / B$. Therefore, near the flow merging region, the location of the maximum $v^{\prime}$ agrees with that of the maximum $\partial T / \partial Y$ and the turbulent heat flux $\overline{v t}$ is produced efficiently in the thermal mixing layer. As a result, the turbulent mixing of hot and cold flows becomes quite active, and the development of the thermal mixing layer is promoted.

In the downstream region, the location of the maximum $v^{\prime}$ disagrees with that of $\partial T / \partial Y$. This means that large $v^{\prime}$ produced around the separation bubble does not contribute effectively to the production of $\overline{v t}$. In particular, in the upper part of the thermal mixing layer, both $v^{\prime}$ and $\partial T / \partial Y$ are so small that the production of $\overline{v t}$ becomes rather inactive. This is the reason why the upward development of the thermal mixing layer stagnates in the downstream region. In the lower part of the mixing layer, on the other hand, $v^{\prime}$ shows very large values around the separation bubble even though $\partial T / \partial Y$ is rather small. Thus, the production of $\overline{v t}$ becomes rather active there in comparison with the upper part, and the thermal mixing layer can develop downward. This causes such an asymmetric development of the thermal mixing layer in the $Y$-direction as observed in Fig. 11. For the discussion on the detailed mixing process of hot and cold air, the turbulent heat fluxes should be measured in the thermal mixing layer, and this is the problem for our future work.

In the development of the HVAC unit, downsizing is one of the most important problems, and the promotion of the mixing of hot and cold air is a key issue to achieve it. In the present study, it has been revealed that the stagnation of the development of the thermal mixing layer is attributed to the disagreement of locations of the maximum $v^{\prime}$ and the maximum $\partial T / \partial Y$. This means that the thermal mixing can be promoted effectively if large $v^{\prime}$ can be produced inside the thermal mixing layer in which $\partial T / \partial Y$ shows large values. The development of the turbulence promoters is also an important problem to improve the performance of the HVAC unit.

\section{Conclusion}

Experimental study has been conducted to make clear the three-dimensional structure of the flow field in the mixing T-junction and examine its influence on the mean temperature distributions of the mixed flow. The influences of the aspect ratio of the channel and the velocity ratio of hot and cold flows have been examined in detail. The main results can be summarized as follows.

(1) The flow entering from the branch is separated at the downstream edge of the T-junction, and a large separation bubble is formed in the main channel. The reverse flow region has a three-dimensional shape with a longer trailing edge at the location near the spanwise centerline of the main channel.

(2) Qualitatively similar distributions of the secondary flow appear in the channels with different $A R$. That is, a longitudinal vortex is formed near the lower corner at $X / B=2$ in which the reverse flow region most develops in the $Y$-direction. In the further downstream region, the longitudinal vortex disappears together with the reattachment of the separated flow.

( 3 ) The fluctuating velocity $v^{\prime}$ that contributes directly to the turbulent transport of heat shows the local maximum in the mixing layer just after the flow merging. It shows a uniform distribution in the spanwise direction there. In the downstream region, $v^{\prime}$ shows large values in the shear layer around the separation bubble, and it increases in the region nearer the spanwise centerline reflecting the three-dimensional feature of the separation bubble.

(4) The mean temperature in the mixed flow shows uniform distributions in the spanwise direction, and thus the influence of the three-dimensional flow field does not appear in it. In the downstream region, the location of the maximum $\partial T / \partial Y$ does not agree with that of the maximum $v^{\prime}$, meaning that the strong turbulence produced around the reverse flow region does not contribute to the turbulent mixing of hot and cold air.

\section{Acknowledgments}

The authors express their thanks to Mr. Naoyasu Shiraki, research engineer of Nagoya University, for his assistance in manufacturing the experimental apparatus.

\section{References}

( 1 ) Kitada, M., Asano, H., Kanbara, M. and Akaike, S., Development of Automotive Air-Conditioning System Basic Performance Simulator: CFD Technique Development, JSAE Review, Vol.21 (2000), pp.91-96. 
( 2 ) Kelso, R.M., Lim, T.T. and Perry, A.E., An Experimental Study of Round Jets in Cross-Flow, J. Fluid Mech., Vol.306 (1996), pp.111-144.

( 3 ) Bruecker, C., Study of the Three-Dimensional Flow in a T-Junction Using a Dual-Scanning Method for ThreeDimensional Scanning Particle Image Velocimetry (3D SPIV), Experimental Thermal Fluid Science, Vol.14 (1997), pp.35-44.

(4) Kawamura, T., Shiina, K., Ohtsuka, M., Tanaka, I., Hirayama, H., Tanimoto, K., Fukuda, T., Sakashita, A., Mizutani, J., Minami, Y., Moriya, S. and Madarame, H., Experimental Study on Thermal Striping in a Mixing Tee with Hot and Cold Water, Proc. 10th Int. Conf. Nuclear Eng., ICONE10-22214, (2002).

( 5 ) Fukushima, N., Fukagata, K., Kasagi, N., Noguchi, H. and Tanimoto, K., Numerical and Experimental Study on Turbulent Thermal Mixing in a T-Junction Flow, 6th ASME-JSME Joint Thermal Eng. Conf., TED-AJ03582, (2003) in CD-ROM.
( 6 ) Wu, H.L., Peng, X.F. and Chen, T.K., Influence of Sleeve Tube on the Flow and Heat Transfer Behavior at a T-Junction, Int. J. Heat Mass Transfer, Vol.46 (2003), pp.2637-2644.

( 7 ) Fukazawa, K. and Sano, M., Study on Turbulent Channel Flow with Injection through an Inclined Slit (Influence of Injection Flow Rate), Trans. Jpn. Soc. Mech. Eng., (in Japanese), Vol.71, No.701, B (2005), pp.3037.

( 8 ) Yoshida, S., Obi, S. and Masuda, S., Organized Vortex Motion in Periodically Perturbed Turbulent Separated Flow over a Back-Ward Facing Step, Int. J. Heat Fluid Flow, Vol.22 (2001), pp.301-307.

( 9 ) Sudo, K., Sumida, M. and Hibara, H., Experimental Investigation on Turbulent Flow in a SquareSectioned 90-Degree Bend, Exp. in Fluids, Vol.30 (2001), pp.246-252. 